\title{
The occurrence and significance of triploidy in the liver fluke, Fasciola hepatica
}

\author{
H. L. FLETCHER*, E. M. HOEY, N. ORR†, A. TRUDGET'T, I. FAIRWEATHER \\ and M. W. ROBINSON:
}

Parasite Proteomics and Therapeutics Research Group, The Queen's University of Belfast, School of Biology and Biochemistry, Medical Biology Centre, 97 Lisburn Road, Belfast BT9 7BL, UK

(Received 7 Fune 2003; revised 11 August 2003; accepted 11 August 2003)

\section{SUMMARY}

Karyotyping of Fasciola hepatica samples from Britain and Ireland has identified a triploid isolate which is effectively aspermic, rendering it necessarily asexually reproducing. Considering the extensive presence of asexually reproducing diploid and triploid Fasciola in Asia it is suggested that facultative gynogenesis is widespread in this parasite. This has important implications for the population genetics and evolution of Fasciola, especially in relation to the development and spread of drug resistance, and must be considered in the mathematical modelling of this process.

Key words: Fasciola hepatica, triploidy, karyotype, parthenogenesis, drug resistance.

\section{INTRODUCTION}

The liver fluke Fasciola hepatica is the most economically important trematode parasite in temperate regions, where cattle and sheep serve as its definitive hosts. The related fasciolid Fasciola gigantica occupies the comparable ecological niche in tropical regions, infecting cattle and water buffalo. Control of fasciolosis in the developed world is generally through the use of anthelminthics; however, there have been several reports of resistance to the most commonly used anthelminthic, triclabendazole (TCBZ), a member of the benzimidazole group of drugs (Overend \& Bowen, 1995; Moll et al. 2000). In order to combat the spread of drug resistance it is desirable to develop predictive models of fluke populations and genetic change under selection. This requires knowledge of Fasciola population genetics and breeding systems.

Parasitic trematodes are hermaphrodite and Fasciola is apparently self-fertile because single flukes can produce eggs (Lagrange \& Gutmann, 1961); however, the high level of heterozygosity in Fasciola from Japan, Korea, USA and Australia (Agatsuma et al. 1994) is indicative of low levels of self fertilization and requires an outbreeding system. Presumably sperm are physically transferred between flukes by copulation using the cirrus. Many

\footnotetext{
* Corresponding author. Tel: +44 2890 272084. Fax: +44 2890 335877. E-mail: h.fletcher@qub.ac.uk

$\dagger$ Present address: Department of Haematology, 'C' Floor, Belfast City Hospital, 51 Lisburn Road, Belfast BT9 7AB.

\$ Present address: The Queen's University of Belfast, The Dept. of Veterinary Science, Stoney Road, Stormont, Belfast BT4 3SD.
}

fasciolids in Japan and Korea exhibit triploidy, causing aspermy (Itagaki et al. 1998; Terasaki et al. 2000). Japanese and Korean diploid strains are also meiotically dysfunctional and aspermic (Agatsuma et al. 1994; Terasaki et al. 1982, 2001) and, like the triploids, cannot self-fertilize and are presumably gynogenic. Facultative gynogenesis has also been inferred to occur in unfertilized ova of Fasciola hepatica (Sanderson, 1958). Thus, it appears that sperm are neither essential for activation of the ovum nor for fertilization, although the viability of eggs produced by different mechanisms is not known. Outbreeding, self-fertilization, gynogenesis and triploidy would influence the recombination of alleles between individuals and hence the degree of genetic diversity generated by each fluke. Local populations (farm or wetland scale) could be outbreeding and diverse or clonal with few genotypes present. This will affect the evolutionary progression of drug resistance. Therefore, $F$. hepatica isolates from the $\mathrm{UK}$ and Ireland have been karyotyped in order to determine their reproductive mode.

\section{MATERIALS AND METHODS}

\section{Fasciola hepatica sources}

Three sources of $F$. hepatica were used. (1) A wild type (TCBZ-susceptible) isolate originally from eggs from bile ducts of sheep slaughtered in an abattoir in Cullompton, Devon, UK, in 1998, and subsequently passed through 2 generations in sheep and 1 in rats. (2) A TCBZ-resistant isolate, originally eggs collected from sheep in Sligo, Ireland, in 1998, subsequently passed through 1 generation in sheep and 2 in rats. Both were provided as metacercariae by 
the Veterinary Laboratory Agency, Weybridge. They were raised in male albino Sprague-Dawley rats. Passaging in rats has used approximately 100 eggs from 3-5 flukes. (3) Wild-type flukes were obtained from cattle livers in a Northern Ireland abattoir.

\section{Cytological preparations}

Transverse slices of fluke approximately $1 \mathrm{~mm}$ thick were cut posterior to the ventral sucker and were incubated in $0.025 \%(\mathrm{w} / \mathrm{v})$ colchicine in isotonic phosphate-buffered saline (PBS), $\mathrm{pH} 7 \cdot 4$ for $1.5 \mathrm{~h}$. Material was either used immediately or after fixation and storage in 3 parts absolute ethanol, 1 part acetic acid at $4{ }^{\circ} \mathrm{C}$. Fragments containing testicular material were used for aceto-orcein squash preparations. They were macerated in $3 \%(\mathrm{w} / \mathrm{v})$ orcein in $45 \%(\mathrm{v} / \mathrm{v})$ acetic acid and squashed under a coverslip. Alternatively, smear preparations were made by rubbing the cut surface of a fresh fluke over a microscope slide, air-drying and staining with orcein. Bright field microscopy was used for observations.

RESULTS

Ten Cullompton isolate flukes and 5 Sligo TCBZresistant flukes from laboratory rats, and 15 flukes from naturally infected cattle were examined. All the Cullompton flukes were found to be triploid $(3 n=30)$, whereas the TCBZ-resistant flukes and local wild-type flukes were all diploid $(2 n=20)$. There were clear gross differences in spermatogenesis between the triploid and diploid flukes. The diploids showed many cells in meiotic metaphase-I containing 10 bivalents and clear chiasmata (Fig. 1A and $\mathrm{B})$. No countable meiotic metaphases were found in triploids but well-spread cells showed 30 chromosomes (Fig. 1C and D). In less well-spread preparations it was apparent that the triploids failed in meiosis. In diploids, the 8-cell clusters passed quickly through both meiotic divisions to give a cluster of 32 spermatids (Fig. 2A). Eight-cell clusters of triploid early prophase I spermatocytes were frequent and appeared to be normal, but a later stage showed highly abnormal first meiotic metaphases with clumps of sticky chromosomes (Fig. 2B). This may suggest some synapsis, but the presence or extent of synaptonemal complexes in Cullompton triploids is not known. The 8 cells attempted divisions to 16 cells, producing variously sized nuclei with some interconnections (Fig. 2B), indicating gross missegregation. These cells were presumably reabsorbed and no later stages were seen. The failure of the triploids to produce spermatozoa was consistent with expectations for an odd-numbered polyploid and similar to observations of Asian triploids (Terasaki et al. 2000).

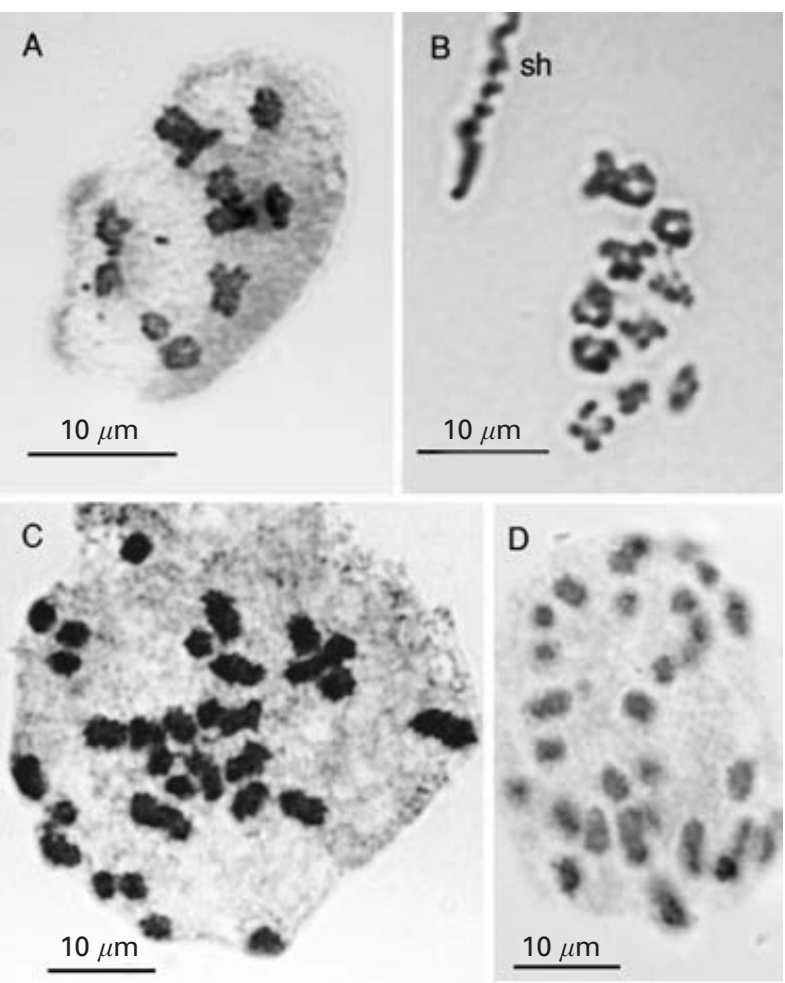

Fig. 1. Meiotic metaphase in Fasciola hepatica. Diploids show 10 bivalents and chiasmata (A and B) and triploids show 30 single chromosomes (C and D). Sh, sperm head.

\section{ISCUSSION}

\section{Occurrence of triploids}

Triploid Fasciola have only been reported previously from Japan (where they predominate), Korea and China (reviewed by Terasaki et al. 2000). The occurrence of triploid Fasciola in the UK shows that triploidy is not purely an Asian phenomenon. The Cullompton isolate is a population originating from many eggs from multiply infected sheep. We cannot determine exactly when the Cullompton isolate became triploid, nor whether the whole isolate was or is triploid. All specimens karyotyped from July 2001 to June 2003 have been triploid. Others not specifically karyotyped were aspermic.

The fertility of aspermic triploids requires gynogenic reproduction. Most odd-number polyploids are practically sterile because meiosis divides by 2 , leaving a remainder of single chromosomes for random distribution. Production of balanced gametes requires all sets of 3 chromosomes (trivalents) to assume the same orientation, a single chromosome proceeding to one pole to produce a haploid secondary spermatocyte, while the other is diploid. Assuming pairing, then whatever orientation the first trivalent takes, there is a probability of 0.5 that each other trivalent will assume the same alignment. Therefore, random segregation of 10 trivalents would produce 2 haploid spermatids in $512\left(=0 \cdot 5^{9}\right)$ meiotic divisions, or $1 / 1024$ spermatids. Failure 


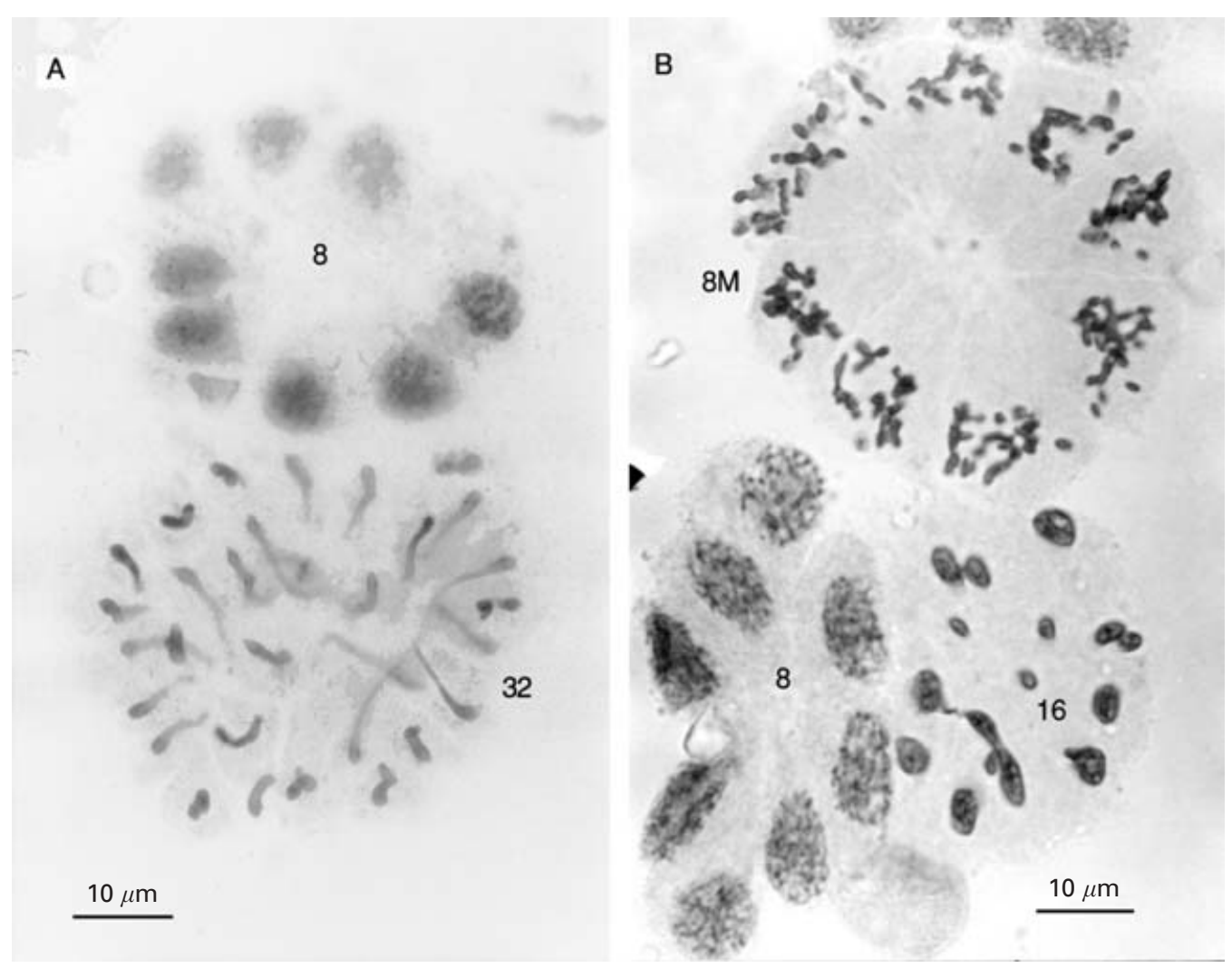

Fig. 2. Spermatogenesis in Fasciola hepatica. (A) In diploids the 8-cell primary spermatocyte rosettes progress through 2 meiotic divisions to produce a syncytium containing 32 spermatids (32). (B) In triploids, the 8-cell prophase primary spermatocyte rosettes (8) appear normal but accumulate at an abnormal metaphase (8M), then progress through abnormal first division to produce 16 secondary spermatocytes with unequal nuclei as a result of missegregation (16).

of synapsis further reduces the chances of producing balanced gametes. Benazzi Lentati (1966, 1970) recounts numerous cases where spermatogonia of polyploid planarians (Dugesia species) can expel 1 set of chromosomes from triploids, or 2 sets from tetraploids, to restore diploidy and produce haploid sperm. The process does not appear to be very efficient. There is no evidence for chromosome elimination happening routinely in Fasciola, but it might explain diploid/triploid mosaics.

The apparently normal fertility of triploids with very few sperm and of aspermic Korean and Japanese diploids (Agatsuma et al. 1994; Terasaki et al. 2001) show that sperm entry is not necessary to activate the eggs, and sperm may be entirely redundant in Fasciola.

\section{Origin of triploidy}

The most likely origin of triploid embryos is the fusion of a sperm nucleus with an unreduced female gamete or with the diploid nucleus of a parthenogenetic egg (Terasaki et al. 2000), although dispermy in a haploid ovum has not been excluded. Triploidy could then be transmitted stably by parthenogenesis. Three different triploid genotypes have been identified in Japan (Agatsuma et al. 1994), indicating at least 3 origins of triploidy there. It seems likely that triploidy arises occasionally and can persist because it does not cause sterility in individuals that are already facultatively parthenogenetic.

Around 5\% (Moriyama, Tsuji \& Seto, 1979, Sakaguchi, 1980) to $20 \%$ (Rhee, Eun \& Lee, 1987) of Japanese and Korean flukes are mixoploid, that is, mosaics containing both diploid $2 n$ and triploid $3 n$ cells. This condition cannot pass through a singlecelled stage such as an egg, so must arise afresh in each generation. Mosaic embryos and miracidia might be explained if a sperm nucleus fused with a diploid nucleus in an early embryo, at the 2- or 4-cell stage, but mosaicism could not pass to adult flukes if individual rediae and cercariae originate as single cells as generally described (e.g. Andrews, 1999). The simplest explanation is that triploid cells can lose a set of chromosomes, generating diploid cells, in the manner described for various Dugesia species by Benazzi Lentati (1966, 1970). Mosaics then do not represent new origins of triploidy, which remains a rare event. It would be interesting to know if mosaicism in flukes is confined to germ cells.

\section{Advantages of triploidy}

The relative selective advantages of triploidy and diploidy could be important. Triploids tend to be larger (Terasaki et al. 2000). Considering the 
example of drug resistance, triploids have an extra allele available, conferring a 50\% higher initial mutation rate per cell. Mutations to detoxify or excrete drugs will be effective immediately (genetically dominant) and more frequent in triploids. Some mutations (e.g. producing tubulin which does not bind benzimidazoles) will have a lower quantitative effect in triploids than in diploids because of dilution by products of the two remaining alleles conferring susceptibility. Polyploids normally have more difficulty becoming homozygous for quantitative mutations. In clonally reproducing parthenogenetic species that avoid meiosis there is no creation of homozygosity for recessive resistance alleles by fusion of genetically identical gametes, so parthenogenetic diploids have no advantage here. On the other hand, selfing hermaphrodite diploids and gynogens fusing meiotic products will achieve homozygosity for half their loci each generation, and will be $>99 \%$ homozygous within 5 generations. Among parthenogenetic lineages, the triploids have more targets for homogenization by allele conversion, but will be slower to lose remaining susceptibility alleles by mutation (e.g. deletion as seen in nematodes; Kwa et al. 1993). Once generated, resistant genotypes are preserved by parthenogenesis, which gives a huge advantage to recessive and quantitative alleles when they are rare (immediately after the first mutation) and in single colonizing and migrating individuals.

Where restricted outbreeding reduces the value of sperm, parthenogens can eliminate male functions and allocate the saved metabolic and spatial resource for female functions, thus increasing egg production compared to hermaphrodites (see Maynard-Smith (1976) for discussion). Both triploids and aspermic diploid Fasciola are still investing in testes and spermatocytes, and thus are not getting the full potential savings of parthenogenesis. The single ootype may limit egg production. Presumably, mutations could occur that stop testis development and then be selected for by the reduction in costs of male functions. Completely asexual reproduction is generally regarded as useful for highly specialized species where recombinants are less well adapted, but as a dead end because evolutionary rates are limited without genetic recombination. There is also the inevitable accumulation of deleterious mutations by Muller's ratchet (Muller, 1932). Some Fasciola strains appear to be on this asexual path, particularly the triploids which are committed to asexuality. It is of importance to know the frequencies of bisexual, self-fertilizing and asexual reproduction in $F$. hepatica populations.

\section{REFERENCES}

AGATSUMA, T., TERASAKI, L., YANG, L. \& BLAIR, D. (1994).

Genetic variation in the triploids of Japanese Fasciola species, and relationships with other species in the genus. Fournal of Helminthology 68, 181-186.

ANDrews, S. J. (1999). The life cycle of Fasciola hepatica.

In Fasciolosis (ed. Dalton, J. P.), pp. 1-29. CABI, Oxon.

BENAZZI lentati, G. (1966). Amphimixis and pseudogamy in freshwater triclads: experimental reconstitution of polyploid pseudogamic biotypes. Chromosoma (Berl.) 20, 1-14.

BENAZZI LENTATI, G. (1970). Gametogenesis and egg fertilization in planarians. International Review of Cytology 27, 101-179.

ITAGAKI, T., TSUTSUMI, K., ITO, K. \& TSUTSUMi, y. (1998). Taxonomic status of the Japanese Triploid forms of Fasciola: Comparison of mitochondrial ND1 and COI sequences with $F$. hepatica and $F$. gigantica. Fournal of Parasitology 84, 445-448.

KWA, M. S., KOOYMAN, F. N., BOERSEMA, J. H. \& ROOS, M. H. (1993). Effect of selection for benzimidazole resistance in Haemonchus contortus on beta-tubulin isotype 1 and isotype 2 genes. Biochemical and Biophysical Research Communications 191, 413-419.

Lagrange, E. \& Gutmann, A. (1961). Sur l'infestation experimentale de la souris par Fasciola hepatica. Rivista di Parassitologia 22, 93-101.

Maynard-Smith, J. (1976). Evolution of Sex. Cambridge University Press, Cambridge.

MOLL, L., GAASENBEEK, C. P. H., VELLEMA, P. \& BORGSTEEdE, F. H. M. (2000). Resistance of Fasciola hepatica against triclabendazole in cattle and sheep in the Netherlands. Veterinary Parasitology 91, 153-158.

Moriyama, N., TSUji, M. \& SETO, T. (1979). Three karyotypes and their phenotypes of Japanese liver flukes (Fasciola sp). Fapanese Fournal of Parasitology 28, 23-33.

MULLER, H. J. (1932). Some genetic aspects of sex. American Naturalist 66, 118-138.

OVEREND, D. J. \& BOWEN, F. L. (1995). Resistance of Fasciola hepatica to triclabendazole. Australian Veterinary Fournal 72, 275-276.

RHEE, J. K., EUN, G. S. \& LEE, S. B. (1987). Karyotype of Fasciola sp. obtained from Korean cattle. The Korean Fournal of Parasitology 25, 37-44.

SAKAGUCHI, Y. (1980). Karyotype and gametogenesis of the common liver fluke, Fasciola sp., in Japan. Fapanese Fournal of Parasitology 29, 507-513.

SANDERSON, A. R. (1958). Maturation and fertilization in two digenetic trematodes, Haplometra cylindracea (Zeder 1800) and Fasciola hepatica (L.). Proceedings of the Royal Society Edinburgh 67, 1958-1959.

TERASAKI, K., AKAHANE, H., HABE, S. \& MORIYAMA, N. (1982). The geographical distribution of common liver flukes (the genus Fasciola) with normal and abnormal spermatogenesis. Fapanese Fournal of Veterinary Science 44, 223-231.

TERASAKI, K., NODA, Y., SHIBAHARA, T. \& ITAGAKI, T. (2000). Morphological comparisons and hypotheses on the origin of polyploids in parthenogenetic Fasciola sp. Fournal of Parasitology 86, 724-729.

TERASAKI, K., ITAGAKI, T., SHIBAHARA, T., NODA, Y. \& MORIYAMA-GONDA, N. (2001). Comparative study of the reproductive organs of Fasciola groups by optical microscope. Fournal of Veterinary Medical Science 63, $735-742$. 
Reproduced with permission of the copyright owner. Further reproduction prohibited without permission. 